\title{
On Isomorphism Theorems for MI-groups
}

\author{
Michal Holčapek, Michaela Wrublová, Martin Štěpnička \\ Centre of Excellence IT4Innovations, division of University of Ostrava \\ Institute for Research and Applications of Fuzzy Modeling \\ 30. dubna 22, 70103 Ostrava 1, Czech Republic \\ \{michal.holcapek, michaela.wrublova, martin.stepnicka\}@osu.cz
}

\begin{abstract}
The theory of MI-algebras ("Many Identities"algebras) has been introduced by M. Holčapek and M. Šťpnička recently. These structures motivated by an algebraic formalizations of distinct arithmetics of fuzzy numbers, generalize the standard structures (monoids, groups, fields etc.) by employing a whole set of identity-like elements called pseudoidentitites. This leads to natural questions: which properties of classical algebraic structures related to arithmetics of crisp numbers are preserved also in the case of MI-structures. This paper focuses on MI-groups, particularly on the properties of their homomorphisms. We show that three crucial theorems of isomorphism that are valid in the classical case, are under some conditions also valid in the theory of MI-groups.
\end{abstract}

Keywords: many identities groups, algebraic structures, fuzzy numbers, isomorphism theorems

\section{Introduction}

The concept of MI-groups was introduced by M. Holčapek and M. Štěpnička in [3, 4] and later on elaborated in [5]. The motivation for the introduction of such algebraic structures came from the arithmetics of fuzzy numbers. It is well-known that standard arithmetics of fuzzy numbers that are based on the Zadeh's extensional principle do lack some classical properties of the arithmetic of crisp numbers. Particularly, the following equalities

(i) $x+(-x)=0$,

(ii) $x x^{-1}=1$,

are not generally satisfied, for $x$ being a fuzzy number and 0, 1 as the crisp zero and unit element, respectively. In other words, the problem lies in the non-existence of inverse elements for both arithmetic operations on fuzzy numbers. But obviously, this is just the consequence of the crispness of both neutral (identity) elements. However, we also know that in the arithmetics of fuzzy numbers:

$$
x+(-x)=\tilde{0}, \quad x x^{-1}=\tilde{1},
$$

where $\tilde{0}, \tilde{1}$ are fuzzy numbers that are located close to 0 and 1 and also from the algebraic point of view, they work similarly to 0 and 1 , respectively.
This led the authors in $[3,4]$ to introduced MIalgebras (e.g. MI-monoids, MI-groups, MI-rings or MI-fields) that naturally generalize standard algebraic structures (monoids, groups, rings or fields) in such a way that they employ so called set of pseudoidentities, i.e., a set of such elements that preserve some but not all properties of classical identities.

It should be noted that the arithmetic of intervals as special fuzzy sets or extended intervals involving infinite intervals has been intensively investigated by Markov [7]. The interesting arithmetic of stochastic intervals can be found in [8].

In this paper, we focus only of MI-groups as a generalization of one of the most natural algebraic structures. We investigate whether the same or similar concepts, theorems and properties, that are known for groups, hold also in the case of MIgroups. The structure of the paper is as follows. Assuming that readers are familiar with the classical theory of groups we only refer to a relevant source of detailed information [6] and for the sake of brevity, omit any introduction to this theory. We recall the basic definitions, propositions and theorems related to MI-groups. Then we describe the concept of homomorphism of MI-groups, the introduce full MI-subgroups, normal full MI-subgroups and quotient MI-groups. Finally, we state conditions under which the three isomorphism theorems are valid.

\section{MI-groups}

MI-group is an algebraic structure based on a generalization of concept of monoid satisfying the cancellation law which is endowed with a monoidal isomorphism representing the inversion. As we have mentioned above the main idea of our generalization of groups consists in the introduction of a set of pseudoidentities which possess similar properties like the identity (neutral) element. As a consequence of the definition we obtain that groups as well as commutative monoids equipped with an involutive operation representing inversion proposed by Bica in [1] (see [2]) form special subclasses of MI-groups.

In what follows, we briefly recall basic definitions and properties used in this paper. For details including an extensive motivation and examples, we refer to $[3,4,5]$. 


\subsection{Basic definitions}

Definition 1. A triplet $(G, \circ, E)$ is said to be an MI-monoid if $E$ is a non-empty subset of $G$ and $\circ$ is a binary operation on $G$ such that for any $x, y, z \in$ $G$ and $a, b \in E$ the following conditions are satisfied:

$(M 1) x \circ(y \circ z)=(x \circ y) \circ z$,

(M2) $\exists e \in E, \forall x \in G: x \circ e=e \circ x=x$,

(M3) $a \circ b \in E$,

(M4) $x \circ a=a \circ x$.

Elements from $E$ are called pseudoidentity elements (pseudoidentities, for short) and a pseudoidentity $e$ satisfying (M2) is called a (strong) identity element. An MI-monoid $G$ is said to be abelian or commutative if $x \circ y=y \circ x$ holds for any $x, y \in G$.

One can see that each MI-monoid $(G, \circ, E)$ is a monoid $(G, \circ)$ with an abelian submonoid $E$ (characterizing pseudoidentity elements) which elements commute with each element of $G$. Each monoid $(G, \circ)$ is an MI-monoid $(G, \circ,\{e\})$, where $e$ is the identity, therefore, MI-monoids generalize monoids.

The concept of MI-monoidal homomorphism is defined in a common manner.

Definition 2. Let $G$ and $H$ be MI-monoids. A mapping $f: G \rightarrow H$ is a homomorphism of $M I$ monoids provided

(HM1) $f(x y)=f(x) f(y)$ for all $x, y \in G$,

(HM2) $f\left(e_{G}\right)=e_{H}$,

(HM3) $f(x) \in E_{H}$ for all $x \in E_{G}$,

where $e_{G}$ and $e_{H}\left(E_{G}\right.$ and $\left.E_{H}\right)$ denote the strong identity (the set of strong identity and pseudoidentities elements) of $G$ and $H$, respectively. If $f$ is injective, $f$ is said to be a monomorphism. If $f$ is surjective, $f$ is said to be a epimorphism. If $f$ is bijective and $f\left(E_{G}\right)=\left\{f(a) \mid a \in E_{G}\right\}=E_{H}, f$ is said to be an isomorphism. In this case $G$ and $H$ are said to be isomorphic (written $G \cong H$ ). $A$ homomorphism $f: G \rightarrow G$ is called an endomorphism and an isomorphism $f: G \rightarrow G$ is called an automorphism.

Definition 3. A MI-monoid G satisfies the cancellation law if

$$
x y=z y \quad \text { implies } \quad x=z
$$

holds for any $x, y, z \in G$

In contrast to the group structure, where the inverse elements are introduced internally, our definition needs an external unary operation defining the inverse elements. In [2], the inverse elements are defined using an involutive monoidal automorphism. Since we deal here with MI-monoids being not abelian in general, we consider an MI-monoidal isomorphism of MI-monoid onto its dual which only reverses the order of operands.
Definition 4. Let $G=(G, \circ, E)$ be an MI-monoid. $A$ dual MI-monoid to $G$ is $G^{o p}=\left(G, \circ^{o p}, E\right)$, where $\circ^{o p}$ is given by $x \circ^{o p} y=y \circ x$.

Definition 5. An MI-monoidal isomorphism $f$ of $G$ onto $G^{o p}$ that satisfies

(G1) $f(x) \circ x \in E$,

(G2) $f(x) \circ x=x \circ f(x)$,

(G3) $f(f(x))=x$

for any $x \in G$ is called an f-inversion (inversion, for short) in $G$.

For the sake of simplicity and conventional manner, the inversion will be denoted using $f(x)=x^{-1}$. An element $x \in G$ with $x^{-1}=x$ is said to be $s y m$ metric in $G$. In the group theory, the symmetric elements are called the elements of the second order, i.e., it holds $x x=e$.

Theorem 1. Let $P_{G}$ be the least submonoid of $G$ containing the set $\left\{x x^{-1} \mid x \in G\right\}$. Then $P_{G}$ is an abelian submonoid of symmetric elements of the monoid $(G, \circ)$.

Sketch of the proof: Put $X=\left\{x x^{-1} \mid x \in G\right\}$ and define $P_{G}=\left\{a_{1} a_{2} \cdots a_{n} \mid a_{i} \in X, n \in N\right\}$, where $N$ denotes the set of all natural numbers. It is easy to verify that $P_{G}$ is the least submonoid of $G$ containing $X$.

The set $P_{G}$ could be interpreted as the least admissible set of pseudoidentities containing "active pseudoidentities", it means that they are the product of an element and its inversion in $G$. Now, we can proceed to introduce the concept of MI-groups.

Definition 6. An MI-monoid endowed with inversion satisfying the cancellation law is called an MIgroup.

It should be noted that the algebraic structure for fuzzy numbers defined by Bica in [1] is an abelian MI-group with $E$ defined as the set of all symmetric elemets of $G$. Note that not all symmetric elements in $G$ are contained in $P_{G}$. It means that Bica's definition is a special example of MI-groups. Another example shows a relationship between MIgroups and fuzzy groups assuming that the lattice operations satisfy the cancellation law.

Example 1. Let us recall that a $\star$-fuzzy subgroup of a group $(G, \circ, e)$ is a fuzzy set $H: G \rightarrow[0,1]$ satisfying the following axioms ([9]):

(i) $H(x) \star H(y) \leq H(x \circ y)$ for any $x, y \in G$,

(ii) $H(x) \leq H\left(x^{-1}\right)$ for any $x \in G$,

(iii) $H(e)=1$, 
where $\star$ is usually the minimum operation, but also left-continuous t-norms are permitted. Define a subset $\mathbb{H}$ of $G \times(0,1]$, a binary operation $\bullet$ and an inversion $^{-1}$ on $\mathbb{H}$ as follows:

$$
\begin{gathered}
(x, \alpha) \in \mathbb{H} \text { iff } \alpha \leq H(x), \\
(x, \alpha) \bullet(y, \beta)=(x \circ y, \alpha \star \beta), \\
(x, \alpha)^{-1}=\left(x^{-1}, \alpha\right) .
\end{gathered}
$$

Put $\mathbb{E}=\{(e, \alpha) \mid \alpha \in(0,1]\}$. One can check that if $\star$ satisfies the cancellation law, then $(\mathbb{H}, \bullet, \mathbb{E})$ is an MI-group. Note that the assumption of cancellation law is important here, otherwise, we need not be able to prove that $\mathbb{E}$ is closed under $\bullet$ and the cancellation law for $\mathbb{H}$ is satisfied.

On the other side, if $G$ is a group and an MIgroup $(\mathbb{H}, \bullet,-1)$ is defined in such a way that $\mathbb{H} \subseteq$ $G \times(0,1]$ such that $(x, \alpha) \in \mathbb{H}$ implies $(x, \beta) \in \mathbb{H}$ for any $\beta \leq \alpha$, and $^{-1}$ are defined as above with respect to the operation of $G$, then

$$
H(x)=\bigvee_{(x, \alpha) \in \mathbb{H}} \alpha
$$

defines $a \star$-fuzzy subgroup of $G$.

\subsection{MI-subgroups}

To investigate the isomorphism theorems for MIgroups, we need a suitable concept of MI-subgroup. The following definition straightforwardly generalizes the definition of subgroup.

Definition 7. Let $G=(G, \circ, E)$ be an MI-group, $H \subseteq G$ and $F \subseteq E$ be non-empty sets. If $H=$ $(H, \circ, F)$ is itself $M I$-group under the product and the inversion of $G$, then $H$ is said to be an MIsubgroup of $G$. This is denoted by $H \leq G$.

In this paper, we restrict ourselves to the socalled canonical MI-subgroups where the set of pseudoidentities $F$ in the MI-subgroup $(H, \circ, F)$ is introduced as the maximal subset of original pseudoidentities in $H$.

Definition 8. An MI-subgroup $(H, \circ, F)$ of an $M I$ group $(G, \circ, E)$ is said to be canonical if $F=H \cap E$.

The following propositions belong among the standard ones in group theory.

Theorem 2. Let $G=(G, \circ, E)$ be an MI-group and $H \subseteq G$ be a non-empty subset. Then, $H$ is a canonical MI-subgroup of $G$ if and only if $e \in H$ and $x y^{-1} \in H$ for all $x, y \in H$.

Sketch of the proof: $(\Rightarrow)$ Trivial. $(\Leftarrow)$ The proof consists in a technical yet exhaustive verification of MI-group axioms.

A typical example of canonical MI-subgroup of $G$ is $P_{G}$.

Theorem 3. Let $G$ be an MI-group. Then $P_{G}$ is a canonical MI-subgroup of $G$.
Sketch of the proof: It is easy to verify that $e_{G} \in$ $P_{G}$ and $x y^{-1} \in P_{G}$ for any $x, y \in P_{G}$.

Theorem 4. Let $G$ be an MI-group and $\left\{H_{i} \mid i \in\right.$ $I\}$ be non-empty set of canonical MI-subgroups of $G$. Then, $\bigcap_{i \in I} H_{i}$ is a canonical MI-subgroup of $G$.

Proof: It is easy to see that $e, x y^{-1} \in \bigcap_{i \in I} H_{i}$ for any $x, y \in \bigcap_{i \in I} H_{i}$. By Theorem $2, \bigcap_{i \in I} H_{i}$ is a canonical MI-group of $\mathrm{G}$.

It is well known that the union of subgroups is not a group in general. Therefore, the least subgroup containing this union is used to define a join of subgroups. The same idea is considered for the union of MI-subgroups.

Definition 9. Let $H, K$ be canonical MI-subgroups of $G$. The least canonical MI-subgroup of $G$ which contains $H \cup K$ is called a canonical join of $H$ and $K$ and is denoted by $H \sqcup K$.

\subsection{Homomorphism MI-groups}

A homomorphism of MI-groups is an MI-monoidal homomorphism preserving the inversion and pseudoidentities. The precise definition is as follows.

Definition 10. Let $G$ and $H$ be $M I$-groups. A mapping $f: G \rightarrow H$ is a homomorphism of MI-groups if

(HG1) $f(x y)=f(x) f(y)$ for all $x, y \in G$,

(HG2) $f(x) \in E_{H}$ for all $x \in E_{G}$,

(HG3) $f\left(x^{-1}\right)=f(x)^{-1}$ for all $x \in G$,

(HG4) if $f(x) \in P_{H}$ for some $x \in G$, then there is $s \in P_{G}$ such that $f(x)=f(s)$,

where $E_{G}$ and $E_{H}$ denote the set of all pseudoidentities of $G$ and $H$, respectively.

Monomorphism, epimorphism and isomorphism of MI-groups are defined equivalently as for MImonoids. Recall that $f\left(E_{G}\right)=E_{H}$ must be satisfied, whenever $f$ is an isomorphism. The generalization of concepts like the kernel or image of a homomorphism of grups is as follows.

Definition 11. The MI-kernel of $f$ it the set MI-Ker $f=\left\{x \in G \mid f(x) \in P_{H}\right\}$. If $A$ is a subset of $G$, then $f(A)=\{y \in H \mid y=$ $f(x)$ for some $x \in A\}$ is the image of $A . f(G)$ is called the image of $f$ and denoted by $\operatorname{Im} f$. If $B$ is a subset of $H$, then $f^{-1}(B)=\{x \in G \mid f(x) \in B\}$ is the inverse image of $B$.

The following theorem shows how the neutral element and the set $P_{G}$ of (active) pseudoidentities elements are preserved with respect to an MI-group homomorphism.

Theorem 5. Let $f: G \rightarrow H$ be a homomorphism of MI-groups. Then, 
(i) $f\left(P_{G}\right) \subseteq P_{H}$.

(ii) $f\left(e_{G}\right)=e_{H}$.

Proof: (i) Let $a \in P_{G}$. Then $a=x_{1} x_{1}^{-1} \cdots x_{n} x_{n}^{-1}$ and $f(a)=f\left(x_{1}\right) f\left(x_{1}\right)^{-1} \cdots f\left(x_{n}\right) f\left(x_{n}\right)^{-1} \in P_{H}$.

(ii) If $x \in G$, then $f\left(x e_{G}\right)=f\left(e_{G}\right) f(x)=$ $e_{H} f(x)$. By the cancellation law, which holds in $H$, we obtain $f\left(e_{G}\right)=e_{H}$.

As a consequence of the previous theorem is the fact that each MI-group homomorphism is also MImonoidal homomorphism.

The following theorem belongs among the folklore in group theory and specifies the monomorphism and isomorphism of MI-groups in terms of MIkernels and compositions of two homomorphisms, respectively.

Theorem 6. Let $f: G \rightarrow H$ be a homomorphism of MI-groups. Then,

(i) $f$ is a monomorphism if and only if MI-Ker $f=P_{G}$ and $f$ restricted to $P_{G}$ is an injective mapping,

(ii) $f$ is an isomorphism if and only if there is a homomorphism $f^{-1}: H \rightarrow G$ such that $f f^{-1}=1_{H}$ and $f^{-1} f=1_{G}$.

Sketch of the proof: (i) $\Rightarrow)$ Obviously, $f$ restricted to $P_{G}$ is injective and $P_{G} \subseteq$ MI-Ker $f$, since $f\left(P_{G}\right) \subseteq P_{H}$. If $x \in$ MI-Ker $f$, then $f(x) \in P_{H}$ and $f(s)=f(x)$ for some $s \in P_{G}$ by (HG4.) Since $f$ is injective, $s=x$ and $x \in P_{G}$, therefore, MI-Ker $f \subseteq P_{G}$.

$(\Leftarrow)$ If $f(x)=f(y)$ for some $x, y \in G$, then $f\left(x y^{-1}\right)=f(x) f(y)^{-1}=f(y) f(y)^{-1} \in P_{H}$. Since MI-Ker $f=P_{G}$, then there exists $t \in P_{G}$ such that $t=x y^{-1}$. Put $r=y^{-1} y \in P_{G}$. Then, $x r=x y^{-1} y=t y=y t$, which implies $f(x) f(r)=$ $f(x r)=f(y t)=f(y) f(t)$. Since $f(x)=f(y)$, we obtain $f(r)=f(t)$ by the cancellation law. Using the assumption on the injectivity of $f$ restricted to $P_{G}$, we obtain $r=t$. Hence, $x r=y t=y r$ and, by the cancellation law, we obtain $x=y$, which implies the injectivity of $f$.

(ii) $\Rightarrow)$ Similarly to the proof in the case of groups, one can simply check that $f^{-1}$ satisfies (HG1)-(HG3). The satisfaction of (HG4) is a consequence of $f\left(P_{G}\right)=P_{H}$.

$(\Leftarrow)$ If $f^{-1}: H \rightarrow G$ is a homomorphism such that $f f^{-1}=1_{G}$ and $f^{-1} f=1_{H}$, then $f$ is a bijection. Now, by the definition of isomorphism of MIgroups, it is sufficient to prove that $f\left(E_{G}\right)=E_{H}$.

The image of a homomorphism is a typical example of canonical MI-subroups.

Theorem 7. If $f: G \rightarrow H$ is a homomorphism, then $\operatorname{Im} f$ is a canonical MI-subgroup of $H$.
Proof: According to Theorem 2, we have to check that $e_{H} \in \operatorname{Im} f$ and $x y^{-1} \in \operatorname{Im} f$ for any $x, y \in \operatorname{Im} f$. By (ii) of Theorem $6, f\left(e_{G}\right)=e_{H} \in \operatorname{Im} f$. If $x, y \in$ $\operatorname{Im} f$, then there are $a, b \in G$ such that $f(a)=x$ and $f(b)=y$. Hence, $f\left(a b^{-1}\right)=f(a) f(b)^{-1}=x y^{-1} \in$ $\operatorname{Im} f$.

\section{Full MI-subgroups}

In this paper, we restrict our investigation of isomorphism theorems to a special subclass of normal MI-subgroups which are called full MI-subgroups. This subclass consists of MI-subgroups which could be characterized as the most similar structure to the classical subgroups because it imitates the properties of subgroups. For example, if $H$ is a subgroup of a group $G$ then $H$ as a subset of $G$ which is closed in the following sense. Note that the same property is not valid for the MI-groups in general.

Definition 12. Let $G$ be an MI-group and $H \subseteq G$ be a non-empty subset. The set $H$ is said to be closed in $G$ if $x s \in H$ for $x \in G$ and $s \in P_{G}$ implies $x \in H$. The set

$$
\bar{H}=\bigcap\{K \subseteq G \mid K \text { is closed and } H \subseteq K\}
$$

is called a closure of $H$ in $G$.

The closure of a subset can be expressed directly as the following theorem demonstrates.

Theorem 8. Let $G$ be an MI-group and $H$ be a non-empty subset of $G$. Then, $\bar{H}=\{x \in G \mid \exists s \in$ $\left.P_{G}: x s \in H\right\}$.

Proof: Obvious.

Now we can approach to the definition of full MIsubgroups.

Definition 13. An MI-subgroup $H$ of an MI-group $G$ is said to be full and denoted by $H \leq_{f} G$ if

(i) $H$ is a canonical MI-subgroup of $G$,

(ii) $H$ is a closed set in $G$, and

(iii) $P_{G} \subseteq H$.

The following theorem shows that the closure of a canonical MI-subgroup is a canonical MI-subgroup which can be used for the construction of full MIsubgroups.

Theorem 9. Let $H$ be a canonical MI-subgroup of $G$. Then, $\bar{H}$ is again a canonical MI-subgroup of $G$.

Proof: Obviously, $e \in \bar{H}$. If $x, y \in \bar{H}$, then $x s, y^{-1} t \in H$ for suitable $s, t \in P_{G}$, which implies $x s y^{-1} t=x y^{-1} s t \in H$, therefore, $x y^{-1} \in \bar{H}$. By Theorem 2, $\bar{H}$ is a canonical MI-subgroup of $G$.

A typical example of full MI-subgroup is the canonically defined MI-subgroup from the closure of MI-kernel of a homomorphism of MI-groups. 
Theorem 10. Let $f: G \rightarrow H$ be a homomorphism of MI-groups. Then, $\overline{\text { MI-Ker } f} \leq_{f} G$.

Proof: By Theorem 9, it is sufficient to show that MI-Ker $f$ is a canonical MI-subgroup of $G$. Obviously, $e_{G} \in \operatorname{MI}-\operatorname{Ker} f$. If $x, y \in \operatorname{MI}-\operatorname{Ker} f$, then $f(x), f(y) \in P_{H}$. By Theorems 2 and 3, we have $f(x)(f(y))^{-1} \in P_{H}$ which implies $x y^{-1} \in$ MI-Ker $f$. Hence, MI-Ker $f$ is a canonical MI-subgroup of $G$ by Theorem 2.

To introduce a similar concept to the normal subgroups in this new settings we need a suitable generalization of (left, right) congruence.

Definition 14. Let $H$ be a full MI-subgroup of an $M I$-group $G$ and $x, y \in G$. We say that $x$ is right congruent to $y$ modulo $H$ denoted $x \equiv_{r} y(\bmod H)$ if there exists $s \in P_{G}$ such that $x s y^{-1} \in H$. We say that $x$ is left congruent to $y$ modulo $H$ denoted $x \equiv_{l} y(\bmod H)$ if there exists $s \in p_{G}$ such that $x^{-1} s y \in H$.

It is easy to check that if $x \equiv_{r} y$, then $x c \equiv_{r} y c$ for any $c \in G$ and analogously for the left congruence. Indeed, if $c \in G$ and $x \equiv_{r} y$, then there is $s \in P_{G}$ such that $x s y^{-1} \in H$. Since $P_{G} \subseteq H$ and $c c^{-1} \in P_{G}$, it holds $x s y^{-1} c c^{-1} \in H$. Using the axiom (M4), $x s y^{-1} c c^{-1}=x s c c^{-1} y^{-1}=x c s(y c)^{-1}$, therefore, $x c s(y c)^{-1} \in H$ and $x c \equiv_{r} y c$.

Theorem 11. Let $H \leq_{f} G$.

(i) Right (resp. left) congruence modulo $H$ is an equivalence relation on $G$.

(ii) The equivalence class of $x \in G$ under right (resp. left) congruence modulo $H$ is the closure of $H x=\{h x \mid h \in H\}$, (resp. $x H=$ $\{x h \mid h \in H\})$ in $G$.

(iii) $|\overline{H x}|=|H|=|\overline{x H}|$.

Sketch of the proof: We will write $x \equiv y$ for $x \equiv_{r}$ $y(\bmod H)$ and prove the theorem for the right congruence and the closures of the right cosets.

(i) The equivalence can be proved similarly to the case of groups with the help of the axiom (M4).

(ii) Put $\langle x\rangle=\{y \in G \mid y \equiv x\}$ and $H x=\{h x \mid$ $h \in H\}$. One can simply check that $\langle x\rangle=\overline{H x}$. by

(iii) Let us define $f: H \rightarrow \overline{H x}$ and $g: \overline{H x} \rightarrow H$

$$
\begin{aligned}
& f(y)=y x \quad \text { for all } y \in \bar{H}, \\
& g(y)=y x^{-1} \quad \text { for all } y \in \overline{H x} .
\end{aligned}
$$

It is easy to see that $f$ and $g$ are one-to-one mappings, which implies that $|H| \leq|\overline{H x}|$ and $|\overline{H x}| \leq$ $|H|$, therefore, $|H|=|\overline{H x}|$.

In the next part, the following denotation is used. If $H \leq_{f} G$ and $x \in G$, then a right coset of $H$ in $G$ is the set $H x$ and a left coset of $H$ in $G$ is the set $x H$.
Corollary 12. Let $H \leq_{f} G$.

(i) $G$ is the union of the closures of the right (left) cosets of $H$ in $G$.

(ii) Two closures of right (left) cosets of $H$ in $G$ are either disjoint or equal.

(iii) For all $x, y \in G, \overline{H x}=\overline{H y}(\overline{x H}=\overline{y H})$ if and only if $x y^{-1} \in H\left(x^{-1} y \in H\right)$.

(iv) If $\mathcal{R}$ is the set of the closures of all right cosets of $H$ in $G$ and $\mathcal{L}$ is the set of the closures of all left cosets of $H$ in $G$, then $|\mathcal{R}|=|\mathcal{L}|$.

\section{Normal MI-subgroup}

In this part, we restrict ourselves to full MIsubgroups and introduce the concept of normal MIsubgroup which is intimately related to homomorphisms similarly as in the case of normal subgroups.

Let us start with a useful lemma. Define $H K=$ $\{h k \mid h \in H \& k \in K\}$ for arbitrary subsets of an MI-group $G$.

Lemma 13. Let $H$ and $K$ be a non-empty subsets of an MI-group G. Then,

$$
\overline{K \bar{H}}=\overline{K H}=\overline{\bar{K} H} .
$$

Proof: We will prove the first equality. The second one can be demonstrated using same arguments. Since $H \subseteq \bar{H}$, it holds $K H \subseteq K \bar{H}$ and $\overline{K H} \subseteq \overline{K \bar{H}}$. If $x \in \overline{K \bar{H}}$, then $x s \in K \bar{H}$ for some $s \in P_{G}$. Therefore, $x s=k h$ for some $k \in K$ and $h \in \bar{H}$. Since $h \in \bar{H}$, there exists $t \in P_{G}$ such that $h t \in H$. Therefore, $x s t=k h t \in K H$ and $x \in \overline{K H}$, since $s t \in P_{G}$.

Let us proceed with a statement that belongs to the folklore in group theory and that characterizes the properties of normal MI-subgroups.

Theorem 14. Let $H \leq_{f} G$. Then the following conditions are equivalent

(i) the left and right congruence modulo $H$ coincide;

(ii) the closure of each left coset of $H$ in $G$ is the closure of a right coset of $H$ in $G$;

(iii) $\overline{x H}=\overline{H x}$ for all $x \in G$;

(iv) $\overline{x H x^{-1}} \subseteq H$ for all $x \in G$;

(v) $\overline{x H x^{-1}}=H$ for all $x \in G$.

Proof: $(i) \Leftrightarrow$ (iii) Obviously, two equivalence relations $R$ and $S$ are identical if and only if the equivalence class of each element under $R$ coincides with its equivalence class under $S$. In this case the equivalence classes are the closures of left and right cosets with respect to $H$. 
(ii) $\Rightarrow($ iii) If $\overline{x H}=\overline{H y}$ for some $y \in G$, then $x \in$ $\overline{H x} \cap \overline{H y}$ which implies $\overline{H x}=\overline{H y}$, since two right cosets are either equal or disjoint. Hence, $\overline{x H}=$ $\overline{H y}=\overline{H x}$.

(iii) $\Rightarrow($ ii $)$ is immediate.

$($ iii $) \Rightarrow($ iv $)$ If $y \in \overline{x H x^{-1}}$, then $y s=x h x^{-1}$ for some $s \in P_{G}$ and $h \in H$. Since $\overline{x H}=\overline{H x}$, then $x h \in \overline{H x}$ which implies $x h t=h^{\prime} x$ for some $t \in P_{G}$ and $h^{\prime} \in H$. Hence, $x h x^{-1} t=x h t x^{-1}=$ $h^{\prime} x x^{-1} \in H$, since $x x^{-1} \in P_{G} \subseteq H$. Therefore, we obtain $y s=x h x^{-1} \in \bar{H}$ and $y \in \bar{H}$ by the closure property. Since $H=\bar{H}$, we obtain $\overline{x H x^{-1}} \subseteq H$.

Similarly, one can prove the implications $(i v) \Rightarrow$ $(v)$ and $(v) \Rightarrow(i i i)$.

Definition 15. A full MI-subgroup $H$ of an $M I$ group $G$ which satisfies the equivalent conditions above is said to be normal MI-subgroup of $G$. We write $H \triangleleft G$ if $H$ is normal in $G$.

An example of normal MI-subgroup of $G$ is the canonical MI-subgroup $\overline{P_{G}}$.

Theorem 15. $\overline{P_{G}} \triangleleft G$.

Proof: By Theorems 3 and $9, \overline{P_{G}}$ is a full MIsubgroup of $G$. By Theorem 14 and Lemma 13, to check the normality of $\overline{P_{G}}$, it is sufficient to verify that $\overline{x \overline{P_{G}}}=\overline{x P_{G}}=\overline{P_{G} x}=\overline{\overline{P_{G}} x}$ holds for any $x \in$ $G$. Let $x \in G$ and $y \in \overline{x P_{G}}$. Then $y s=x t=t x$ for suitable $s, t \in P_{G}$. Hence, ys $\in P_{G} x$, which implies $y \in \overline{P_{G} x}$ and $\overline{x P_{G}} \subseteq \overline{P_{G} x}$. The inverse inclusion also holds, which concludes the proof.

Recall that $H \sqcup K$ denotes the canonical join of MI-subgroups $H$ and $K$ of $G$.

Theorem 16. Let $H \triangleleft G$ and $K \leq_{f} G$. Then,

(i) $H \cap K \triangleleft K$;

(ii) $H \triangleleft H \sqcup K$;

(iii) $\overline{H K}=\overline{H \sqcup K}=\overline{K H}$.

Sketch of the proof: (i) By Theorem $4, H \cap K$ is a canonical MI-subgroup of $G$. Since $H$ and $K$ are both closed in $G, H \cap K$ is closed in $G$. Since $P_{G} \subseteq H \cap K$, we obtain $H \cap K \leq_{f} G$. Let $k \in K$ be arbitrary. If $x \in H \cap K$, then $k x k^{-1} \in K$. Since $H$ is a normal MI-subgroup of $G$, we obtain $k x k^{-1} \in \overline{k H k^{-1}} \subseteq H$ by Theorem 14. Therefore, $k x k^{-1} \in H \cap K$. By the closure property, $\overline{k(H \cap K) k^{-1}} \subseteq \overline{H \cap K}=H \cap K$, and $H \cap K$ is a normal MI-subgroup in $K$.

(ii) It immediately follows from $H \leq_{f} H \sqcup K$.

(iii) Trivially $\overline{H K} \subseteq \overline{H \sqcup K}$. The opposite inclusion can be proved by the expression of elements of $\overline{H \sqcup K}$ by the finite products of elements of $\mathrm{H}$ and $K$. Using the normality of $H$, these products can be expressed in the form $h k s$ for some $h \in H, k \in K$ and $s \in P_{G}$.
The quotient MI-group is defined as a triplet with two non-empty sets and new operation. The elements of quotient MI-group are sets of closures of cosets. The operation on closures of cosets is defined analogously as in the classical theory of group.

Theorem 17. If $H \triangleleft G, G / H$ is the set of closures of all (left) cosets of $H$ in $G$ and $E / H$ is the set of closures of all cosets $x H$ for $x \in E$, then $G / H=(G / H, \star, E / H)$ is an MI-group under the binary operation given by

$$
\overline{x H} \star \overline{y H}=\overline{x y H}
$$

and the inversion given by

$$
(\overline{x H})^{-1}=\overline{x^{-1} H} .
$$

The MI-group $G / H$ is called the quotient MI-group of $G$ by $H$.

Sketch of the proof: The proof consists in the technical yet exhaustive verification of the correctness of defined operations and the MI-group axioms.

The following lemma demonstrates that each quotient MI-group $G / H$ has only one "active" pseudoidentity that is necessary equal to the strong identity element. Therefore, each quotient MI-groups can be in some sense interpreted as a common group (with pseudoidentities that are, however, unimportant from the group perspective).

Lemma 18. . Let $H \triangleleft G$. Then $P_{G / H}=\{H\}$.

Proof: If $x \in G$, then $\overline{x H} \star(\overline{x H})^{-1}=\overline{x H} \star$ $\overline{x^{-1} H}=\overline{x x^{-1} H}=\overline{s H}=H$, since $s \in P_{G} \subseteq H$. Hence, $P_{G / H}=\{H\}$.

The following theorem shows a relationship between normal MI-subgroups and MI-kernels of homomorphisms.

Theorem 19. If $f: G \rightarrow H$ is a homomorphism of MI-groups, then $\overline{\mathrm{MI}-\operatorname{Ker} f} \triangleleft G$. Conversely, if $H \triangleleft G$, then the map $\pi: G \rightarrow G / H$ given by $\pi(x)=$ $\overline{x H}$ is an epimorphism with $H=\overline{\operatorname{MI}-\operatorname{Ker} \pi}$.

Proof: Put $K=\overline{\text { MI-Ker } f}$. By Theorem $10, K$ is a full MI-subgroup of $G$. We will demonstrate that $K$ is normal in G. Put . If $x \in G$ and $k \in K$, then $f\left(x k x^{-1}\right)=f(x) f(k) f(x)^{-1}=f(x) f(x)^{-1} f(k) \in$ $P_{H}$, since $f(x) f(x)^{-1} \in P_{H}$ and $f(k) \in P_{H}$ by the definition of MI-kernel. Therefore, $x k x^{-1} \in K$ and $x K x^{-1} \subseteq K$ for any $x \in G$. Since $K$ is closed, then also $\overline{x K x^{-1}} \subseteq K$ and, by (iv) of Theorem 14, we obtain that $K$ is normal in $G$.

By the definition of the product of closures of left cosets, we obtain $\pi(x y)=\overline{x y H}=\overline{x H} \star \overline{y H}=$ $\pi(x) \star \pi(y)$. If $x \in E$, then $\pi(x)=\overline{x H} \in E / H$. If $x \in G$, then $f\left(x^{-1}\right)=\overline{x^{-1} H}=(\overline{x H})^{-1}=f(x)^{-1}$. The axiom (HG4) immediately follows from the fact that $P_{G / H}=\{H\}$ by Lemma 18 and $\pi\left(e_{G}\right)=H$. 
Hence, $\pi$ is a homomorphism of $G$ to $G / H$, which is clearly surjective. Therefore, $\pi$ is an epimorphism.

Finally, we will prove that $\overline{\mathrm{MI}-\operatorname{Ker} \pi}=H$. If $h \in H$, then $\pi(h)=\overline{h H}=H$, since $h \in H \cap \overline{h H}$ and the closures of two left cosets are either disjoint or equal. Therefore, $H \subseteq \overline{\mathrm{MI}-\operatorname{Ker} \pi}$. If $x \in G$ such that $\pi(x) \in P_{G / H}$, then $\pi(x)=H$ which implies $\overline{x H}=\bar{H}$ and $x \in H$. Therefore, MI-Ker $\pi \subseteq H$, which implies $\overline{\text { MI-Ker } \pi} \subseteq \bar{H}=H$.

Theorem 20. Let $H, K$ be canonical MI-subgroups of $G, H \leq K$ and $H \triangleleft G$. Then, $H \triangleleft K, H \triangleleft \bar{K}$ and $K / H=\bar{K} / H$.

Proof: The first two parts immediately follow from Theorem 9 and the definition of normal MIsubgroups. To demonstrate $K / H=\bar{K} / H$, it is sufficient to prove that $\bar{K} / H \subseteq K / H$, the opposite inclusion follows from $K \subseteq \bar{K}$. Recall that $H$ is full, therefore, $P_{G} \subseteq H$. Now, if $\overline{x H} \in \bar{K} / H, x \in \bar{K}$, then $x s \in K$ for some $s \in P_{G}$. Since also $s \in H$, we obtain $x s \in \overline{x H}$. Therefore, $\overline{x s H}=\overline{x H}$, since two left cosets are either disjoint or equal. Hence, each left coset in $\bar{K} / H$ is also a left coset in $K / H$, i.e., $\bar{K} / H \subseteq K / H$.

\section{Three isomorphism theorems}

To show the main theorem and its consequences we need to introduce a weaker concept of epimorphism called quasi-epimorphism. The quasi-epimorphism is defined with the help of congruence as follows.

Definition 16. A homomorphism $f: G \rightarrow H$ is a quasi-epimorphism if for any $y \in H$ there exists $x \in G$ such that $y \equiv f(x)\left(\bmod \overline{P_{H}}\right)$.

Now, we can provide the main theorem which consequences are the well known isomorphism theorems.

Theorem 21. If $f: G \rightarrow H$ is a homomorphism of $M I$-groups and $K \triangleleft G$ contained in $\overline{\text { MI-Ker } f}$, then there exists a unique homomorphism $\tilde{f}: G / K \rightarrow$ $H / \overline{P_{H}}$ making the following diagram commutative:

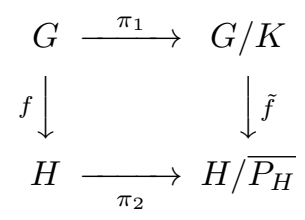

Moreover, $\pi_{2}(\operatorname{Im} f)=\operatorname{Im} \tilde{f} . \quad \overline{\text { MI-Ker }} f / K \subseteq$ MI-Ker $\tilde{f}$. If $\tilde{f}$ is an isomorphism, then $f$ is a quasi-epimorphism and $K=\overline{\mathrm{MI}-K e r} f$. If $f$ is an epimorphism and $K=\overline{\text { MI-Ker } f}$, then $\tilde{f}$ is an isomorphism.

Sketch of the proof: To prove the first statement one has to check that $\tilde{f}: G / K \rightarrow H / \overline{P_{H}}$ given by

$$
\tilde{f}(\overline{x K})=\overline{f(x) P_{H}}=\overline{f(x) \overline{P_{H}}}
$$

for any $\overline{x K} \in G / K$ defines a unique homomorphism making the diagram commutative.

If $\overline{y P_{H}} \in \pi_{2}(\operatorname{Im} f)$, then $\overline{y P_{H}}=\overline{f(x) P_{H}}=\tilde{f}(\overline{x K})$ for some $x \in G$. Therefore, $\overline{y P_{H}} \in \operatorname{Im} \tilde{f}$ and $\pi_{2}(\operatorname{Im} f) \subseteq \operatorname{Im} \tilde{f}$. Similarly, one can check the opposite inclusion.

If $\overline{x K} \in \overline{\text { MI-Ker } f} / K$, where $x \in \overline{\text { MI-Ker } f}$, then $\tilde{f}(\overline{x K})=\overline{f(x) P_{H}}=\overline{P_{H}}$, since $f(x) \in \overline{P_{H}}$. Hence, $\overline{x K} \in$ MI-Ker $\tilde{f}$ and $\overline{\text { MI-Ker } f} / K \subseteq$ MI-Ker $\tilde{f}$.

If $\tilde{f}$ is an isomorphism and $y \in H$, then there exists $x \in G$ such that $\tilde{f}(\overline{x K})=\overline{f(x) P_{H}}=\overline{y P_{H}}$, which implies $f(x) \equiv y\left(\bmod \overline{P_{H}}\right)$. Therefore, $f$ is a quasi-epimorphism. The equality $\overline{\text { MI-Ker } f}=K$ can be simply proved from $P_{G / K}=\{K\}$.

It is easy to see that $\tilde{f}$ is surjective. If $\tilde{f}(\overline{x K})=\tilde{f}(\overline{y K})$ for some $\overline{x K}, \overline{y K} \in G / K$, then, according Corollary 12, $\overline{f(x) P_{H}}=\overline{f(y) P_{H}}$ implies $f(x)^{-1} f(y) s \in P_{H}$ for some $s \in P_{H}$. Since $f$ is an epimorphism, there exists $t \in P_{G}$ such that $f\left(x^{-1} y t\right) \in P_{H}$ and $x^{-1} y t \in$ MI-Ker $f$. Therefore, $x y^{-1} \in \overline{\text { MI-Ker } f}=K$, which implies $\overline{x K}=\overline{y K}$ and $\tilde{f}$ is an injective mapping.

Theorem 22. If one of the following conditions is satisfied

(i) $P_{H}=\overline{P_{H}}$,

(ii) $f$ is an epimorphism,

then $\overline{\mathrm{MI}-\operatorname{Ker} f} / K=$ MI-Ker $\tilde{f}$ holds in the previous theorem.

Proof: Obvious.

Lemma 23. Under the assumptions of Theorem 21, MI-Ker $\tilde{f}$ is closed in $G / K$.

Proof: Let $\overline{x K} \in$ MI-Ker $\tilde{f}$. By Lemma 18, $P_{G / K}=\{K\}$ and $K$ is the strong identity element. Hence, $\overline{x K} \star K=\overline{x K} \in$ MI-Ker $\tilde{f}$.

Corollary 24 (First Isomorphism Theorem). If $f: G \rightarrow H$ is a homomorphism of MI-groups and $P_{H}$ is closed, then $f$ induces an isomorphism $G / \overline{\text { MI-Ker } f} \cong \operatorname{Im} f / \overline{P_{\text {Im } f}}$.

Proof: By Theorem 7, $f: G \rightarrow \operatorname{Im} f$ is an epimorphism of MI-groups. The required isomorphism is a straightforward consequence of the last statement of Theorem 21.

Corollary 25 (Second Isomorphism Theorem). Let $G$ be an MI-group. If $K \leq_{f} G$ and $H \triangleleft G$, then $K /(H \cap K) \cong H K / H$.

Proof: By Theorems 16 and 20, we can define a homomorphism $f: K \rightarrow H K / H$ as a composition $K \hookrightarrow \overline{H \sqcup K}=\overline{H K} \rightarrow \overline{H K} / H=H K / H$ where $\hookrightarrow$ is an inclusion of homomorphism. It easy to check that $\overline{\mathrm{MI}-\operatorname{Ker} f}=H \cap K$. By the previous corollary, $K / \overline{\mathrm{MI}-\operatorname{Ker} f}=K /(H \cap K) \cong$ 
$\operatorname{Im} f \overline{P_{\operatorname{Im} f}}$. Since $\operatorname{Im} f=H K / H$, we obtain $\operatorname{Im} f / \overline{P_{\operatorname{Im} f}}=(H K / H) / \overline{P_{(H K / H)}} \cong H K / H$, where clearly $\overline{P_{\operatorname{Im} f}}=\overline{P_{(H K / H)}}=\{H\}$ and $G / P_{G} \cong G$, whenever $P_{G}$ contains just the strong identity element.

Corollary 26 (Third Isomorphism Theorem). Let $G$ be an MI-group. If $H \triangleleft G$ and $K \triangleleft G$ such that $K \leq H$, then $H / K \triangleleft G / K$ and $(G / K) /(H / K) \cong$ $G / H$.

Proof: Let $\pi: G \rightarrow G / H$ be the canonical epimorphism. Since $\overline{\text { MI-Ker } \pi}=H$ by Theorem $19, K \triangleleft \overline{\text { MI-Ker } \pi}=H$ by Theorem 16 and $(G / H) / \overline{P_{G / H}} \cong G / H$, there exists a unique epimomorphism $\tilde{\pi}: G / K \rightarrow G / H$ with $\overline{\text { MI-Ker } \pi} / K=$ MI-Ker $\tilde{\pi}=H / K$ according to Theorems 21 and 22. By Lemma 23, $\overline{\text { MI-Ker } \tilde{\pi}}=H / K$, therefore, $H / K \triangleleft G / K$ according to Theorem 19 , which concludes the first part of the corollary.

Since $\pi$ is the canonical epimorphism, $\tilde{\pi}$ has to be an epimorphism, i.e., $\operatorname{Im} \tilde{\pi}=G / H$. Moreover, $H / K=\overline{\text { MI-Ker } \tilde{\pi}}$ as was demonstrated above. Then, using Corollary 24 and $\operatorname{Im} \tilde{f}=G / H$, we obtain $(G / K) /(H / K) \cong(G / H) / \overline{P_{G / H}} \cong G / H$, and the proof is finished.

\section{Conclusions}

In this paper, we developed a part of MI-group theory devoted to the crucial isomorphism theorems which are well known in group theory. We repeated the basic notions related to MI-groups and introduced the concepts of normal MI-subgroup as a special full MI-subgroup and quotient MI-group. The isomorphism theorems was proved as special corollaries of more general theorem. For the second and third isomorphism theorem, we had to assume that the set of pseudoidentities contains just symmetric elements.

Since the quotient MI-groups in the setting of full MI-subgroups have the group structure, there is a natural question if the isomorphism theorems are also true for the non-full MI-subgroups. We leave this non-trivial question to our future reseach.

\section{Acknowledgement}

This presentation has been elaborated in the framework of the IT4Innovations Centre of Excellence project, reg. no. CZ.1.05/1.1.00/02.0070 supported by Operational Programme "Research and Development for Innovations" funded by Structural Funds of the European Union and state budget of the Czech Republic.

This work was supported by grant SGS06/PřF/2013 of the University of Ostrava.

\section{References}

[1] A.M. Bica. Categories and algebraic structures for real fuzzy numbers. PU.M.A., Pure Math. Appl., 13(1-2):63-77, 2003.

[2] A.M. Bica. Algebraic structures for fuzzy numbers from categorial point of view. Soft Comput., 11(11):1099-1105, 2007.

[3] M. Holčapek and M. Štěpnička. Arithmetics of extensional fuzzy numbers - part I: Introduction. In Proc. IEEE Int. Conf. on Fuzzy Systems, page submitted, Brisbane, 2012.

[4] M. Holčapek and M. Štěpnička. Arithmetics of extensional fuzzy numbers - part II: Algebraic framework. In Proc. IEEE Int. Conf. on Fuzzy Systems, page submitted, Brisbane, 2012.

[5] M. Holčapek and M. Štěpnička. Mi-algebras: a new framework for arithmetics of (extensional) fuzzy numbers. Submitted to Fuzzy Sets Systems, 2012.

[6] Thomas W. Hungerford. Algebra. New York: Holt, Rinehart and Winston, Inc. XIX, 502 p. , 1974.

[7] S. M. Markov. Extended interval arithmetic involving infinite intervals. Mathematica Balkanica, 6(3):269-304, 1992.

[8] S. M. Markov and R. Alt. Stochastic arithmetic: Addition and multiplication by scalars. Applied Numerical Mathematics, 50:475-488, 2004.

[9] John N. Mordeson and D.S. Malik. Fuzzy commutative algebra. Singapore: World Scientific, 1998. 